\title{
Maternal dietary omega-3 fatty acids and placental function
}

\author{
Megan L Jones, Peter J Mark and Brendan J Waddell \\ School of Anatomy, Physiology and Human Biology, The University of Western Australia, \\ 35 Stirling Highway, Crawley, Perth, Western Australia 6009, Australia \\ Correspondence should be addressed to B J Waddell; Email: brendan.waddell@uwa.edu.au
}

\begin{abstract}
The developing fetus requires substantial amounts of fatty acids to support rapid cellular growth and activity. Although the fatty acid composition delivered to the fetus is largely determined by maternal circulating levels, the placenta preferentially transfers physiologically important long-chain polyunsaturated fatty acids (LC-PUFAs), particularly omega-3 (n-3) PUFAs. Maternal dietary supplementation with n-3 PUFAs during pregnancy has been shown to increase gestation length, enhance fetal growth, and reduce the risk of pregnancy complications, although the precise mechanisms governing these effects remain uncertain. Omega-3 PUFAs are involved in several physiological pathways which could account for these effects, including anti-inflammatory, pro-resolving, and anti-oxidative pathways. Recent studies have shown that maternal dietary n-3 PUFA supplementation during rat pregnancy can reduce placental oxidative damage and increase placental levels of pro-resolving mediators, effects associated with enhanced fetal and placental growth. Because several placental disorders, such as intrauterine growth restriction, preeclampsia, and gestational diabetes mellitus, are associated with heightened placental inflammation and oxidative stress, there is considerable interest in the potential for dietary n-3 PUFAs as a therapeutic intervention for these disorders. In this study, we review the impact of dietary n-3 PUFAs on placental function, with particular focus on placental inflammation, inflammatory resolution, and oxidative stress. Reproduction (2014) 147 R143-R152
\end{abstract}

\section{Introduction}

Fatty acids are important biological constituents with metabolic, structural, and signaling roles. The developing fetus requires substantial amounts of fatty acids to support rapid cellular growth and activity, and among these the omega-3 (n-3) and omega- 6 ( $n-6)$ polyunsaturated fatty acids (PUFAs) are crucial (Haggarty 2010). The most biologically important $n-3$ and $n-6$ PUFAs are eicosapentaenoic acid (EPA; 20:5n-3), docosahexaenoic acid (DHA; 22:6n-3), dihomo gamma linolenic acid (20:3n-6), and arachidonic acid (AA; 20:4n-6) (Haggarty 2010). While these long-chain PUFAs (LC-PUFAs) are metabolic derivatives of the essential fatty acids, $\alpha$-linolenic acid, and linoleic acid (LA), their formation from these precursors is energetically demanding and so they are most readily obtained from the diet (McCowen \& Bistrian 2005).

Anthropological, nutritional, and genetic studies suggest that humans evolved on a diet consisting of $n-6$ and $n-3$ PUFAs in a ratio of around 1:1 (Simopoulos 2011). In contrast, modern dietary trends have led to a relative deficiency in n-3 PUFA consumption, and an n-6 PUFA intake which far exceeds nutritional requirements. Consequently, the n-6:n-3 PUFA ratio of the current
Western diet is $\sim 10-20: 1$, and this is thought to promote the pathogenesis of many prevalent human diseases (Simopoulos 2011). The n-3 PUFA, DHA, appears to be of particular importance to human health, with this fatty acid considered essential to the cerebral expansion in human evolution (Crawford \& Broadhurst 2012). Accordingly, DHA is particularly crucial to fetal and infant neural development (see review, Rogers et al. (2013)).

Placenta-related disorders affect around one-third of pregnancies world-wide (Jauniaux et al. 2006); examples include intrauterine growth restriction (IUGR), preeclampsia (PE), gestational diabetes mellitus (GDM), and many cases of spontaneous miscarriage. Despite the differing pathologies of these disorders, each is associated with placental inflammation and oxidative stress (Burton \& Jauniaux 2011), and possibly disturbed LC-PUFA delivery (Magnusson et al. 2004). Given that n-3 PUFAs exhibit both anti-oxidative and antiinflammatory properties, maternal dietary n-3 PUFA supplementation has been proposed as a potential therapeutic intervention for placenta-related disorders. Indeed, maternal dietary supplementation with $\mathrm{n}-3$ PUFAs during pregnancy can exert beneficial effects including increased gestation length and increased fetal growth (Imhoff-Kunsch et al. 2012, Larqué et al. 2012, 
Jones et al. 2013a), and a reduced risk of pregnancy complications (Oken et al. 2007). In this study, we review the impact of dietary n-3 PUFAs on placental function, with particular focus on placental fatty acid transport, inflammation, inflammatory resolution, oxidative status, and angiogenesis.

\section{Maternal dietary supply}

The enzymes responsible for the synthesis of LC-PUFA from essential fatty acid precursors $(\Delta 5-$ and $\Delta 6$ destaurases) are undetectable (Hanebutt et al. 2008) or expressed at very low levels in the placental tissue (Wadhwani et al. 2013). As such, placental synthesis of LC-PUFAs is unlikely to meet fetal demand, and the fetus must rely on maternal dietary supply and fatty acid stores to access physiologically important LC-PUFAs. We have recently shown that maternal, placental, and fetal fatty acid compositions are highly dependent on maternal dietary fatty acids in rat (Jones et al. 2013a). We also demonstrated that placental and fetal tissue levels of LC-PUFAs are positively correlated with their respective levels in maternal plasma during late gestation (Jones et al. 2013a), similar to observations in human pregnancy near term (Elias \& Innis 2001). These studies highlight the capacity of maternal dietary fatty acids to effectively alter placental and fetal lipid environments.

\section{Placental fatty acid transport}

Adequate LC-PUFA delivery is critical for optimal fetal development. In particular, fetal accretion of DHA is essential for early development of the brain and the visual system (Rogers et al. 2013), and overall demand peaks during the final trimester of human pregnancy to accommodate rapid growth of the fetal brain (Duttaroy 2009). The placenta normally mediates adequate delivery of physiologically important PUFAs to the fetus by extracting and transporting fatty acids in a directional, preferential, and timely manner (Haggarty 2010). In pathological pregnancies (e.g. IUGR and GDM), however, disturbances in the molecular mechanisms governing placental fatty acid transport have been reported (Magnusson et al. 2004).

\section{Placental regulation of fatty acid supply}

The placenta may play a key role in the regulation of fatty acid availability via the release of placental-derived leptin, a potent stimulator of lipolysis (Haggarty 2004). Human placental BeWo trophoblast cells synthesise leptin and secrete it at both the apical and basal surfaces (Wyrwoll et al. 2005). Accordingly, the human placenta secretes leptin into both maternal and fetal circulations, although $>95 \%$ of total leptin is released into the maternal compartment (Linnemann et al. 2000,
Lepercq et al. 2001), potentially acting to mobilize maternal fat stores. A study by Hoggard et al. (2001) has shown that placental leptin secretion increases as fetal:placental weight ratio increases, which as the authors suggest may enhance fatty acid availability as fetal requirement increases during late gestation.

\section{Preferential placental fatty acid uptake and delivery}

Comparisons between maternal and fetal plasma fatty acid levels demonstrate that transport of physiologically important LC-PUFAs occurs in a directional and preferential manner, closely correlating with fetal requirement (Haggarty 2010). This has been demonstrated following oral ingestion of stable isotope-labeled fatty acids by pregnant women $12 \mathrm{~h}$ before elective caesarean, where placental transfer of DHA was preferential to that of palmitic acid (16:0), oleic acid (OA; 18:1n-9), and LA (Gil-Sánchez et al. 2010).

Maternal circulating total fatty acid concentrations increase during late pregnancy (Gil-Sánchez et al. 2011), an effect driven by a shift from lipid storage in early pregnancy to lipid catabolism in late pregnancy, in part due to rising estrogen levels (Duttaroy 2009). This gestational hyperlipidemia enhances placental access to fatty acids. Placental lipoprotein lipase $(\mathrm{LPL})$ and epithelial lipase, which are present in the maternal-facing microvillous membrane of the syncytiotrophoblast (Fig. 1), release fatty acids from maternal circulating tricyglyceride (TG)-rich lipoproteins to allow

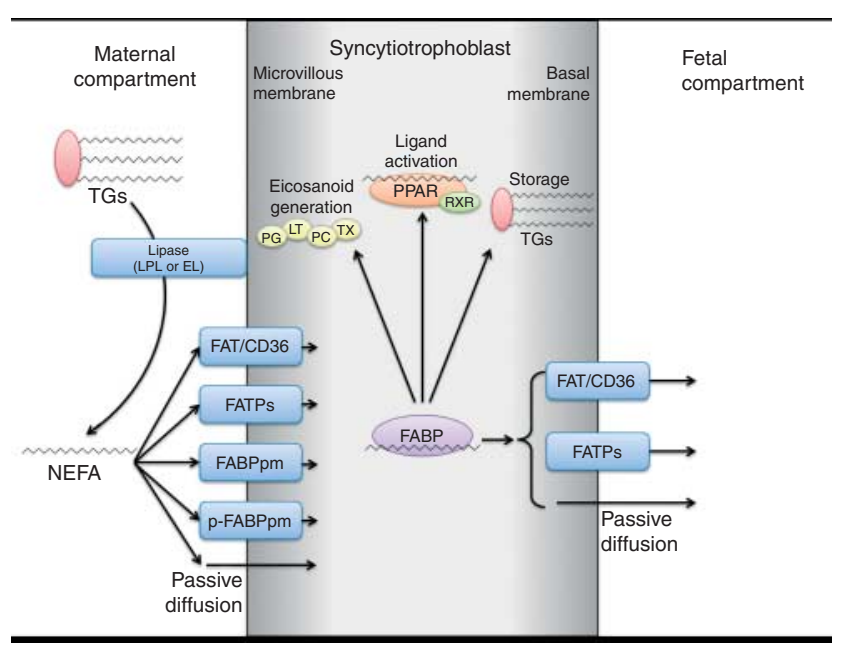

Figure 1 Schematic representation of placental fatty acid transport mechanisms and potential metabolic fates within the placental tissue (modified from Hanebutt et al. (2008)). TGs, tricyglycerides; LPL, lipoprotein lipase; EL, epithelial lipase; NEFA, non-esterified fatty acid; FAT/CD36, fatty acid translocase; FATPs, fatty acid transport proteins; FABP, fatty acid-binding protein; FABPpm, plasma membrane FABP; p-FABPpm, placental plasma membrane FABP; PG, prostaglandin; LT, leukotriene; PC, prostacyclin; TX, thromboxane; PPAR, peroxisome proliferator-activated receptor; $\mathrm{RXR}$, retinoic acid $\mathrm{X}$ receptor. 
placental uptake of non-esterified fatty acids (NEFAs) (Gil-Sánchez et al. 2011). Concentrations of two NEFAs, $\mathrm{DHA}$, and $\mathrm{AA}$ are three- to four-fold higher in the placental intervillous space than in the maternal circulation, at least around the time of delivery (Benassayag et al. 1997), suggesting placental lipases selectively release LC-PUFAs from TGs. Indeed, LPL is known to preferentially hydrolyse fatty acids in the second position of TGs, which tend to be less saturated (Christensen et al. 1995). Placental lipase activity increases during the final trimester of pregnancy, probably serving to enhance placental fatty acid delivery during the period of maximal fetal fatty acid requirement (Duttaroy 2009).

NEFAs may enter the placental syncytium by passive diffusion or via several membrane-bound carrier proteins (Fig. 1) including fatty acid translocase (FAT/CD36), fatty acid transport proteins (FATP1-6), plasma membrane fatty acid-binding protein (FABPpm), or placental plasma membrane FABP (p-FABPpm) (Hanebutt et al. 2008). Although the specific mechanisms of these proteins in placental fatty acid uptake, metabolism, and transfer are not fully understood, directional and preferential transfer of fatty acids across the placenta could be attributable to differences in the affinity of NEFAs for these proteins. For example, p-FABPpm exhibits higher affinity and binding capacity for DHA and AA compared with OA and LA (Campbell et al. 1998a). This carrier protein is also exclusively found in the microvillous membrane of the syncytiotrophoblast, potentially driving unidirectional transfer of LC-PUFAs from maternal to fetal compartments (Campbell et al. 1998b).

Once within the cell, NEFAs bind to cytosolic FABPs which facilitate intracellular fatty acid movement and interactions with subcellular organelles (Hanebutt et al. 2008, Gil-Sánchez et al. 2011). FABPs have increasing affinity for fatty acids with increasing chain length; however, binding affinity is tissue specific (Richieri et al. 2000) and FABP's binding specificity in placental tissue is currently unknown.

\section{Regulation of placental fatty acid transport}

Placental LC-PUFAs appear to act in an autocrine fashion to regulate their own uptake, transport, and metabolism. This is supported by reports showing that n-3 PUFA supplementation increases placental FATP1 and FATP4 mRNA expression in both human and rat (Larqué et al. 2006, Wadhwani et al. 2013), presumably to further enhance LC-PUFA uptake. In addition, incubation of placental BeWo cells with LC-PUFAs (DHA, EPA, and AA) stimulates further cellular LC-PUFA uptake (Johnsen et al. 2009). Accordingly, we have recently reported that $n-3$ PUFA levels in rat placental labyrinth zone (LZ) are proportionally higher in n-3 PUFA-supplemented pregnancies than would be predicted based on control levels (Fig. 2; Jones et al. 2013a),
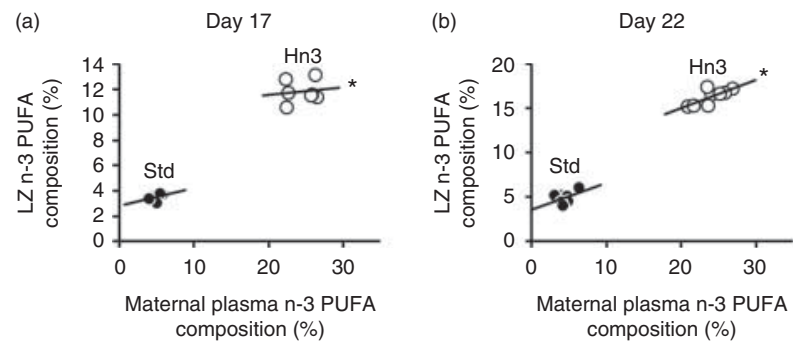

Figure 2 Linear regression analyses for n-3 PUFA composition (\% of total fatty acids) of maternal plasma and placental LZ at (a) day 17 and (b) day 22 of rat pregnancy. This figure was originally published in Jones et al. (2013a). Mothers were fed either a standard (Std) or high n-3 PUFA (Hn3) diet from day 1 of pregnancy. There was no significant difference in the slope of the regression lines between the two diet groups. $* P<0.05$ compared with elevation of regression line for the Std group (ANCOVA).

suggesting placental LZ uptake and retention of $n-3$ PUFAs is enhanced in response to heightened n-3 PUFA availability.

This autocrine effect may be mediated via a number of molecular regulators, including the peroxisome proliferator-activated receptors (PPARs). PPARs are ligand-activated nuclear transcription factors which play critical roles in metabolic, developmental, and inflammatory processes. Many PUFAs, including DHA, EPA, $\mathrm{AA}$, and LA, are natural ligands for PPARs (Jawerbaum \& Capobianco 2011), and all three PPAR isoforms $(\alpha, \beta / \delta$, and $\gamma$ ) are present in the placenta (Hewitt et al. 2006a). Knockout studies indicate that PPAR $\gamma$ and $\beta / \delta$ are particularly important for placental development and vascularization (Jawerbaum \& Capobianco 2011). Once activated, PPARs form heterodimers with retinoic acid $X$ receptor; this complex then binds to the PPAR-response element (PPRE) in the promoter region of target genes to drive their expression. PPREs have been identified in the promoter regions of genes encoding fatty acid transport proteins (Frohnert et al. 1999, Schachtrup et al. 2004). Accordingly, oral supplementation of a PPAR $\gamma$ agonist, rosiglitazone, has been shown to increase placental mRNA expression of Fabppm, Fat (Cd36), Fatp1, and Fatp4 in mice, although Fatp2, Fatp3, and Fatp6 were reduced (Schaiff et al. 2007). Interestingly, placental labyrinthine mRNA expression of Ppara and Pparg increased towards term in rat (Hewitt et al. 2006a), suggesting increased fatty acid trafficking at this time.

\section{Omega-3 fatty acids and placental inflammation}

Inflammation is the immediate response of the innate immune system, which serves to remove invading pathogens and promote regeneration of damaged tissues (Calder 2003). Pregnancy is often described as a state of controlled, mild, maternal, systemic inflammation, as evidenced by higher circulating levels of proinflammatory mediators in pregnant women compared 
with non-pregnant women (Rusterholz et al. 2007). Pregnancy-related inflammation is proposed to play a key role in maternal tolerance of the semi-allogenic fetus (Paulesu et al. 2010), and induce maternal insulin resistance thereby enhancing glucose passage to the developing fetus (Parsons et al. 1992). Although pregnancy-related inflammation is considered adaptive, excessive placental inflammation is associated with a number of pregnancy disorders including preterm birth (Kemp et al. 2010), PE (Rusterholz et al. 2007), miscarriage (Kwak-Kim et al. 2009), IUGR (Amarilyo et al. 2011), and GDM (Kuzmicki et al. 2009).

\section{Differential eicosanoid synthesis}

Eicosanoids are important immunological messengers, which include prostaglandins (PGs), prostacyclins, thromboxanes (TX), and leukotrienes (LT). PGs are important regulators of parturition, with an involvement in promotion of cervical ripening, uterine contractility, regulation of placental blood flow, and fetal adaptations to labor (Keelan et al. 2003, Hertelendy \& Zakár 2004). Eicosanoids, however, are also central to inflammatory activation, and excessive levels are associated with cellular damage.

In response to an inflammatory stimulus, phospholipase enzymes, primarily phospholipase $A_{2}$, release a 20-carbon PUFA (AA or EPA) from the cellular membrane, which is then metabolised either by cyclooxygenase (COX) enzymes to become a PG or TX, or by lipoxygenase- 5 (LOX5) to become an LT (Fig. 3). COX1 is generally considered constitutive, whereas COX2 is rapidly induced by inflammatory stimuli (Calder 2003). Depending on the specific fatty acid derivative, eicosanoids can be either pro- or antiinflammatory. As cellular membranes contain relatively high amounts of the $n-6$ PUFA, AA, this is the principle eicosanoid precursor (Calder 2003). AA-derived eicosanoids are primarily pro-inflammatory; acting to enhance local blood flow; increase pro-inflammatory cytokine; and reactive oxygen species (ROS) production, and bring about fever, erythema, pain, and edema (Calder 2003).

Dietary intake of $n-3$ PUFAs results in the partial replacement of AA by EPA in cellular membranes (Calder 2003). Thus, EPA acts as a competitive inhibitor of AA eicosanoid synthesis by decreasing $\mathrm{AA}$ availability in cellular membranes. In addition, EPA competes with AA for COX- and LOX5-active sites, the resultant eicosanoids which differ in structure and are far less biologically potent (Calder 2011). In contrast to the pro-inflammatory AA-derived eicosanoids, those derived from EPA are either directly anti-inflammatory or pro-inflammatory, but synthesis occurs at low efficiency or not at all (Calder 2003). Consequently, dietary n-3 PUFA supplementation can reduce inflammation by either disrupting proinflammatory eicosanoid generation or promoting the generation of anti-inflammatory forms.

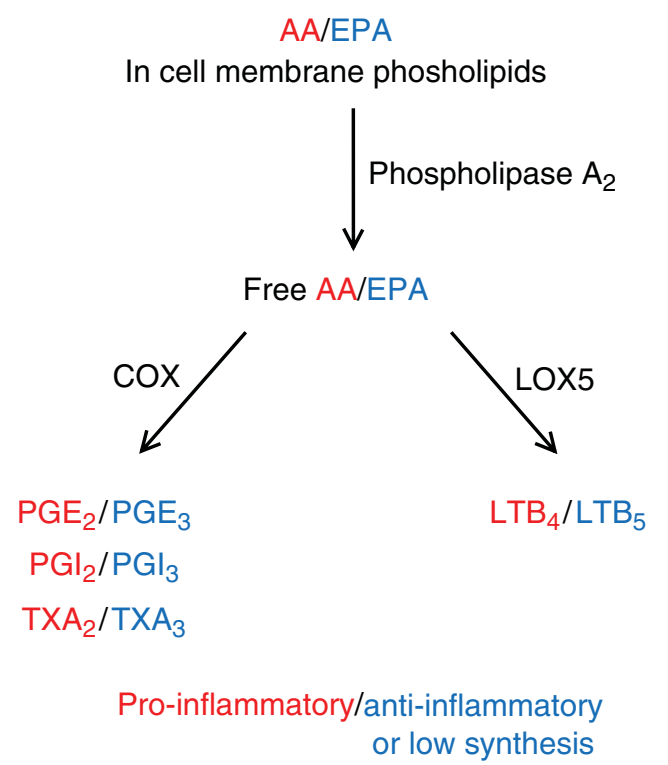

Figure 3 Eicosanoid generation from LC-PUFAs, AA, and EPA. AA-derived eicosanoid are of the 2- or 4-series, and typically promote pro-inflammatory mechanisms. EPA-derived eicosanoids are of the 3- or 5-series, and either exert anti-inflammatory actions, are pro-inflammatory but relatively less potent, or disrupt pro-inflammatory eicosanoid generation by competitively inhibiting production.

During pregnancy, dietary n-3 PUFA supplementation from gestational week 20 has been shown to reduce LT production in neonatal neutrophils by gestational week 37 (Prescott et al. 2007). Similarly, pre-treatment of decidual cells with n-3 PUFAs in vitro decreased PG production, whereas production was enhanced with AA treatment (Arntzen et al. 1998).

\section{Cytokine production}

Cytokines are signaling molecules involved in a wide range of physiological processes, including inflammatory, immunosuppressive, cellular differentiation, and apoptotic pathways (Paulesu et al. 2010). Cytokines also play a central role in reproduction, allowing the maternal-fetal crosstalk required to coordinate the events of a successful pregnancy (Paulesu et al. 2010). During the early stages of pregnancy, the uterine inflammatory environment is characterized by increased pro-inflammatory cytokine production, which is important for embryo receptivity and implantation. Near term, levels of pro-inflammatory cytokines again increase rapidly, which is thought to stimulate the PG synthesis associated with parturition. Besides these two developmental stages, the majority of gestation is characterized by a predominance of anti-inflammatory cytokines allowing for uterine quiescence, and fetal growth and development (Paulesu et al. 2010). Excessive generation of pro-inflammatory cytokines throughout pregnancy is apparent in placental disorders, including recurrent spontaneous abortion and PE (Paulesu et al. 2010). 
The ability of n-3 PUFAs to reduce pro-inflammatory cytokine levels has been demonstrated in rodent and human studies (see review, Calder (2011)). Despite this, surprisingly little is known regarding the impact of n-3 PUFAs on placental pro-inflammatory cytokine levels. Stark et al. (2013) recently demonstrated that DHA reduces lipopolysaccharide (LPS)-induced tumor necrosis factor $\alpha$ levels in term, but not in preterm, placental explants. In contrast, we found that maternal dietary n-3 PUFA supplementation in rat pregnancy did not suppress placental mRNA expression of key pro-inflammatory cytokines near term (Jones et al. 2013b). Interestingly, we also recently demonstrated that glucocorticoids, steroid hormones well recognized for their potent anti-inflammatory effects (Vandevyver et al. 2013), were similarly unable to suppress placental expression of pro-inflammatory cytokines in late gestation in rat (Mark et al. 2013). Collectively, these data suggest that placental inflammatory regulation during late gestation may be unconventional, possibly complicated by signaling pathways that promote parturition.

\section{Fatty acid-activated transcription factors and receptors}

The precise molecular mechanisms governing the anti-inflammatory effects of n-3 PUFAs are uncertain, although it has been proposed that they act to modulate the activity of key transcription factors (PPARs and/or nuclear factor $\kappa \mathrm{B}(\mathrm{NF} \kappa \mathrm{B})$ ) and receptors (i.e. G-protein-coupled receptor (GPR120)) involved in inflammatory signaling.

In addition to the involvement of PPARs in placental fatty acid trafficking described previously, they also mediate the expression of inflammatory genes. For example, activation of PPAR $\gamma$ and/or PPAR $\beta / \delta$ can reduce the expression of $C O X 2$, inducible nitric oxide synthase (iNOS), cellular adhesion molecules, pro-inflammatory cytokines, and $\mathrm{NF} \kappa \mathrm{B}$ target genes (Bensinger \& Tontonoz 2008, Jawerbaum \& Capobianco 2011). Many PUFAs including DHA, EPA, AA, and LA are natural ligands to PPARs (Jawerbaum \& Capobianco 2011), and therefore the anti-inflammatory effects of n-3 PUFAs may be mediated via PPAR activity. Perhaps surprisingly, the effect of dietary n-3 PUFA supplementation on placental PPAR activity is currently unknown.

Recently, membrane-associated GPR120, has been identified and implicated in anti-inflammatory signaling (Oh et al. 2010). Importantly, DHA and EPA, but not AA, palmitic, or myristic acids, promote GPR120-mediated gene activation (Oh et al. 2010). This suggests a unique role of this receptor in the mediation of $n-3$ PUFA-driven anti-inflammatory effects, but the specific role of GPR120 in the placenta has not yet been elucidated.

$\mathrm{NF} K \mathrm{~B}$ is a nuclear transcription factor involved in a range of cellular functions, but is particularly well characterized for its role in inflammation. Upon activation, $\mathrm{NF \kappa B}$ is able to translocate to the nucleus where it promotes the expression of proinflammatory cytokines, adhesion molecules, and iNOS and COX2 genes (Calder 2011). Dietary supplementation with n-3 PUFAs (EPA and DHA) has been shown to suppress NFKB activity in several in vivo and in vitro studies (see review, Calder (2011)).

\section{Omega-3 fatty acids and placental inflammatory resolution}

Until recently, it was thought that the resolution of inflammation was a passive process, simply due to the eventual dissipation of pro-inflammatory signals. It is now clear, however, that inflammatory resolution is a highly active and coordinated process, largely driven by specialized pro-resolving lipid mediators (SPMs; Recchiuti \& Serhan 2012). These SPMs, namely resolvins, protectins, maresins, and lipoxins, are directly derived from LC-PUFAs and are often regarded as specialized forms of eicosanoids due to their common generation by COX and LOX enzymes. Resolvins, protectins, and maresins are derived from n-3 PUFAs; resolvins can be generated from either EPA (E-series resolvins; RvE) or DHA (D-series resolvins; RvD), whereas protectins and maresins are derived only from DHA (Recchiuti \& Serhan 2012). Maresins were only recently identified, and so evidence to confirm their anti-inflammatory and pro-resolving effects are limited. In contrast, the potential biological activity of resolvins and protectins has been confirmed by many in vivo and in vitro studies (for review see, Recchiuti \& Serhan (2012)).

We have recently reported, for the first time, the presence of resolvins, protectins, and their precursors in the rat placental LZ (Jones et al. 2013b). Levels of these SPMs were relatively high in placental tissue, and the level of protectins increased toward term, suggesting an involvement in the maintenance of a healthy inflammatory balance, because pro-inflammatory signals rise markedly leading up to, and during, parturition (Keelan et al. 2003). Maternal dietary n-3 PUFA supplementation effectively increased placental levels of these proresolving mediators (Jones et al. 2013b), potentially enhancing the placental capacity to resolve inflammation. These findings highlight the therapeutic potential of n-3 PUFAs to limit placental inflammation associated with pregnancy disorders, although further functional studies in placental tissues are required.

Lipoxins are derived from the n-6 PUFA, AA, and have shown clear pro-resolving and anti-inflammatory activities in various in vitro and in vivo studies (for review see Ryan \& Godson (2010)). With regard to the placenta, in vitro administration of lipoxin $\mathrm{A}_{4}\left(\mathrm{LXA}_{4}\right)$ reduced LPSinduced apoptosis, pro-inflammatory cytokine secretion, and $N F \kappa B$ activity in human extravillous trophoblast 
cells, and in vivo administration of the synthetic $\mathrm{LXA}_{4}$ analog, BML111, during mid-gestation reduced lowdose LPS-induced placental pro-inflammatory cytokine mRNA expression in rat (Lin et al. 2012). Although these findings signify a potential for AA to promote pro-resolving effects via lipoxin generation, a balance must be met between these pro-resolving mediators and the potentially damaging effects of AA-derived proinflammatory eicosanoids.

\section{Omega-3 fatty acids and placental oxidative stress}

There is mounting evidence that excessive oxidative stress in utero-placental tissues plays a pivotal role in the development of pregnancy complications (Jauniaux et al. 2006, Burton \& Jauniaux 2011). Oxidative stress occurs when cellular production of ROS, by-products of cellular respiration, exceeds the protective capacity of local antioxidant defences and thus causes damage to cellular components. ROS levels are limited by a range of antioxidant enzymes including the superoxide dismutase, catalase, glutathione peroxidase, and/or the thioredoxin system (Burton \& Jauniaux 2011). Alternatively, oxidative damage may be limited by reduced ROS production. For example, it has been proposed that uncoupling proteins (UCPs) limit ROS generation by uncoupling oxidative phosphorylation (Mailloux \& Harper 2011), a primary metabolic source of ROS. Oxidative stress can be exacerbated by inflammation and vice versa, as pro-inflammatory cytokines are produced in response to ROS and subsequently stimulate further ROS production by target cells (Burton \& Jauniaux 2011).

The importance of limiting placental and fetal ROS exposure is highlighted during early pregnancy, while the earliest stages of embryonic and fetal development (approximately the first 10 weeks of human gestation) take place in a low oxygen environment $(<20 \mathrm{mmHg})$ (Jauniaux et al. 2006). This hypoxia is thought to protect the rapidly dividing embryonic and fetal cells from ROSmediated damage, and is achieved by the presence of trophoblastic 'plugs' preventing blood flow into the intervillous space. At $\sim 11-14$ weeks human gestation, these trophoblastic plugs detach, thus increasing blood flow and oxygen tension $(>50 \mathrm{mmHg}$ ) as required to facilitate rapid growth of the fetus (Jauniaux et al. 2006). The onset of placental perfusion is accompanied by a rapid influx of ROS (Jauniaux et al. 2006) and an increased synthesis of enzymatic antioxidants to limit ROS-induced cellular damage (Jauniaux et al. 2000). Accordingly, the premature onset of full maternal-fetal circulation is associated with many miscarriages (Burton \& Jauniaux 2011).

Placental ROS generation is high due to the high inherent metabolic activity of the placental cells; therefore all major antioxidant systems are present within the placenta (Perkins 2006, Jones et al. 2010).
The beneficial effect of limiting placental ROS levels has recently been shown by two separate studies. First, Umekawa et al. (2008) demonstrated that global overexpression of the antioxidant enzyme, thioredoxin-1, in mouse pregnancy reduced placental oxidative status and increased fetal growth. Similarly, we have recently demonstrated that maternal n-3 PUFA dietary supplementation reduced placental levels of $F_{2}$-isoprostanes, a highly reliable marker of oxidative damage (Fig. 4), which was associated with enhanced fetal and placental growth (Jones et al. 2013a). Collectively, these studies suggest that during normal pregnancy, placental ROS may tonically suppress fetal growth.

Omega-3 PUFAs could potentially limit oxidative damage by enhancing ROS scavenging capacity and/or by limiting ROS generation. We recently demonstrated that maternal n-3 PUFA supplementation enhanced mRNA expression and activity of major antioxidant enzymes in the placental LZ across the final third of rat pregnancy (Jones et al. 2013c), coincident with reduced placental oxidative damage (Jones et al. 2013a). These findings are consistent with an in vitro study, in which treatment of placental BeWo cells with modest levels of DHA reduced oxidative DNA damage and increased cell survival when challenged by an oxidative insult (Shoji et al. 2009). Similarly, in human placental explants, DHA administration reduced LPS-induced oxidative damage and restored antioxidant capacity (Stark et al. 2013). Dietary n-3 PUFAs may also reduce ROS generation, given that they increase Ucp2 mRNA expression in mouse white adipose tissue (Hun et al. 1999) and rat myocytes (Hatakeyama \& Scarpace 2001). Importantly, UCP2 has been localized to human

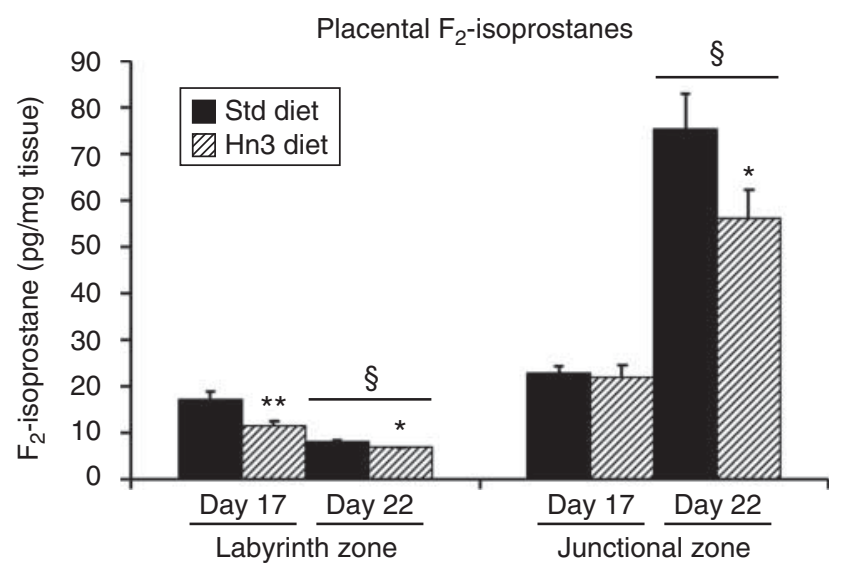

Figure 4 Levels of $\mathrm{F}_{2}$-isoprostanes (pg/mg tissue) in the female placental labyrinth and junctional zones at days 17 and 22 of rat pregnancy. This figure was originally published in Jones et al. (2013a). Mothers were fed either a standard (Std) or high omega-3 ( Hn3) diet from day 1 of pregnancy. Values are mean \pm S.E.M. ( $n=4-8$ per group). ${ }^{*} P<0.05$ and ${ }^{* *} P<0.01$ overall compared with corresponding Std diet group in the same zone (two-way ANOVA; post hoc LSD); ${ }^{\S} P<0.001$ compared with day 17 in the same zone (two-way ANOVA). 
placental tissue, being predominantly expressed by the syncytiotrophoblast (Stark et al. 2012), although maternal dietary n-3 PUFA supplementation in the rat did not affect placental $U_{c p 2}$ mRNA expression in our recent study (Jones et al. 2013c). The effect of maternal n-3 PUFA supplementation on oxidative status of the early gestation placenta, and of particular interest during the onset of placental perfusion, is unknown.

\section{Omega-3 fatty acids and placental angiogenesis}

Omega-3 PUFAs typically inhibit angiogenic pathways, whereas n-6 PUFAs promote angiogenesis. This is supported by several in vitro studies, where n-3 PUFA administration has been shown to reduce formation of capillary-like structures (tubes) in a number of endothelial cell models (reviewed in Massaro et al. (2008)). In vivo evidence is currently limited to tumor-associated angiogenesis, whereby n-3 PUFA administration limits angiogenesis via reduced synthesis of pro-angiogenic AA-derived eicosanoids and transcriptional downregulation of several growth factors (for review see Kang \& Lui (2013)). In the placenta, however, n-3 PUFAs appear to promote rather than inhibit vascular development. This has been demonstrated by in vitro studies, where n-3 PUFA administration in the first trimester placental extravillous trophoblast cell line, HTR8/SVneo, upregulated angiogenic growth factors such as vascular endothelial growth factor-A (VEGF-A) and angiopoietin-like 4, which was associated with enhanced tubular network formation (Johnsen et al. 2011, Basak \& Duttaroy 2013a,b). Interestingly, recent studies have suggested that in first trimester trophoblastic cells, n-3 PUFA-stimulated tube formation was associated with increased expression of FABP4 associated with enhanced tube formation (Basak \& Duttaroy 2013a,b). Whether FABP4 directly mediates the placental proangiogenic response or simply reflects n-3 PUFA-driven fatty acid trafficking is unclear. Regardless, proangiogenic effects of $n-3$ PUFAs in the placenta may in part facilitate the enhanced fetal and placental growth observed in dietary n-3 PUFA-supplemented pregnant rats (Jones et al. 2013a). It is important to note, however, that possible angiogenic effects of $n-3$ PUFAs on the placenta are yet to be investigated in an in vivo model.

Interestingly, as placental Pparg expression increases over late gestation in rat (Hewitt et al. 2006a), expression of the angiogenic factor Vegfa similarly increases (Hewitt et al. 2006b). Accordingly, downregulation of placental Pparg expression by dexamethasone treatment (Hewitt et al. 2006a) is associated with reduced Vegfa expression and fetal capillary density in the placenta, indicating reduced placental angiogenesis (Hewitt et al. 2006b). Therefore, n-3 PUFA-stimulated activation of PPAR $\gamma$ may be a key mediator of placental angiogenesis.

\section{Potential risks of omega-3 dietary supplementation}

Although there are potential benefits associated with dietary n-3 PUFA supplementation during pregnancy, it is also important to ascertain the potential risks, especially given that many women currently increase n-3 PUFA intake during gestation. It has been suggested that excessive dietary n-3 PUFA intake may exacerbate cellular damage caused by an oxidative insult. This is based on the susceptibility of n-3 PUFAs to lipid peroxidation, the by-products of which can damage cellular components (Al-Gubory 2012). An in vitro study on placental cells by Shoji et al. (2009) demonstrated enhanced lipid peroxidation and reduced cell survival in response to high levels of DHA administration $(100 \mu \mathrm{M})$, although modest levels $(1$ or $10 \mu \mathrm{M})$ reduced DNA oxidative damage and enhanced cell survival rate. This was recently supported by Stark et al. (2013), who found high doses of DHA administration $(100 \mu \mathrm{M})$ in term placental explants enhanced lipid peroxidation and DNA oxidative damage, while more modest doses (1 or $10 \mu \mathrm{M})$ reduced LPS-induced oxidative damage and restored antioxidant capacity. Our in vivo research has found that despite relatively high dietary n-3 PUFA supplementation in pregnant rats, placental levels of the ROS-induced lipid peroxidation marker, $\mathrm{F}_{2}$-isoprostanes, were reduced (Fig. 4; Jones et al. 2013a). In contrast, Franke et al. (2010) have recently shown marginally higher plasma lipid peroxidation with n-3 PUFA supplementation in pregnant women, although the placental contribution to these enhanced levels is unknown. Further research is required to clarify the potential risks associated with enhanced dietary n-3 PUFA supplementation.

\section{Concluding remarks}

Overall, results from our research and others suggest that maternal dietary n-3 PUFA supplementation may provide a therapeutic intervention to limit placental inflammation and oxidative stress associated with several pregnancy disorders. Despite this, clinical trials of n-3 PUFA supplementation demonstrate conflicting results regarding pregnancy outcome (Imhoff-Kunsch et al. 2012, Larqué et al. 2012). It has been suggested that this is because supplementation is often begun during mid to late gestation ( 22 weeks; Larqué et al. 2012). Maternal adipose tissue composition contributes significantly to fetal fatty acid accretion during late gestation. Therefore, small changes in the composition of habitual maternal diet before pregnancy, or during early pregnancy, are likely to have a large effect on LC-PUFA delivery to the fetus during late gestation (Haggarty 2004). Importantly, where our group has seen enhanced placental levels of pro-resolving mediators and reduced placental oxidative damage associated with maternal dietary n-3 PUFAs, supplementation began on day 1 of 
rat pregnancy (Jones et al. 2013a,b). Perhaps surprisingly, the possible beneficial effects of early dietary n-3 PUFA supplementation on placental function in humans are unknown. Although recognizing the potential benefits of n-3 PUFA intake during pregnancy, the World Health Organization states that evidence is as yet insufficient to support routine supplementation with fish oil during pregnancy (World Health Organization 12.13.2011). The studies reviewed here provide a strong basis for investigation into the effects of n-3 PUFA supplementation from early pregnancy on placental function, with particular regard to inflammatory and oxidative status, in a clinical setting. It is also important to note that the large majority of studies in this area have each considered only one time period, generally in the second half of gestation, and so there is a clear need for additional studies on the effects of $n-3$ PUFAs on placental function at earlier stages of pregnancy.

\section{Declaration of interest}

The authors declare that there is no conflict of interest that could be perceived as prejudicing the impartiality of the review.

\section{Funding}

This review did not receive any specific grant from any funding agency in the public, commercial or not-for-profit sector.

\section{References}

Al-Gubory KH 2012 Mitochondria: omega-3 in the route of mitochondrial reactive oxygen species. International Journal of Biochemistry \& Cell Biology 44 1569-1573. (doi:10.1016/j.biocel.2012.06.003)

Amarilyo G, Oren A, Mimouni FB, Ochshorn Y, Deutsch V \& Mandel D 2011 Increased cord serum inflammatory markers in small-for-gestational-age neonates. Journal of Perinatology 31 30-32. (doi:10.1038/jp.2010.53)

Arntzen KJ, Brekke OL, Vatten L \& Austgulen R 1998 Reduced production of PGE2 and PGF2 $\alpha$ from decidual cell cultures supplemented with $n-3$ polyunsaturated fatty acids. Prostaglandins \& Other Lipid Mediators 56 183-195. (doi:10.1016/S0090-6980(98)00048-3)

Basak S \& Duttaroy AK 2013 a cis-9,trans-11 conjugated linoleic acid stimulates expression of angiopoietin like-4 in the placental extravillous trophoblast cells. Biochimica et Biophysica Acta 1831 834-843. (doi:10.1016/j.bbalip.2013.01.012)

Basak S \& Duttaroy AK 2013 b Effects of fatty acids on angiogenic activity in the placental extravillous trophoblast cells. Prostaglandins, Leukotrienes, and Essential Fatty Acids 88 155-162. (doi:10.1016/j.plefa.2012.10.001)

Benassayag C, Mignot TM, Haourigui M, Civel C, Hassid J, Carbonne B, Nunez EA \& Ferre F 1997 High polyunsaturated fatty acid, thromboxane A2, and $\alpha$-fetoprotein concentrations at the human feto-maternal interface. Journal of Lipid Research 38 276-286.

Bensinger SJ \& Tontonoz P 2008 Integration of metabolism and inflammation by lipid-activated nuclear receptors. Nature 454 470-477. (doi:10.1038/ nature07202)

Burton GJ \& Jauniaux E 2011 Oxidative stress. Best Practice \& Research. Clinical Obstetrics \& Gynaecology 25 287-299. (doi:10.1016/ j.bpobgyn.2010.10.016)

Calder PC 2003 N-3 polyunsaturated fatty acids and inflammation: from molecular biology to the clinic. Lipids 38 343-352. (doi:10.1007/ s11745-003-1068-y)
Calder PC 2011 Fatty acids and inflammation: the cutting edge between food and pharma. European Journal of Pharmacology 668 (Supplement 1) S50-S58. (doi:10.1016/j.ejphar.2011.05.085)

Campbell FM, Gordon MJ \& Duttaroy AK 1998a Placental membrane fatty acid-binding protein preferentially binds arachidonic and docosahexanoic acids. Life Sciences 63 235-240. (doi:10.1016/S0024-3205 (98)00267-7)

Campbell FM, Bush PG, Veerkamp JH \& Duttaroy AK $1998 b$ Detection and cellular localization of plasma membrane-associated and cytoplasmic fatty acid-binding proteins in human placenta. Placenta 19 409-415. (doi:10.1016/S0143-4004(98)90081-9)

Christensen MS, Høy CE, Becker CC \& Redgrave TG 1995 Intestinal absorption and lymphatic transport of eicosapentaenoic (EPA), docosahexaenoic (DHA), and decanoic acids: dependence on intramolecular triacylglycerol structure. American Journal of Clinical Nutrition 61 56-61.

Crawford MA \& Broadhurst CL 2012 The role of docosahexaenoic and the marine food web as determinants of evolution and hominid brain development: the challenge for human sustainability. Nutrition and Health 21 17-39. (doi:10.1177/0260106012437550)

Duttaroy AK 2009 Transport of fatty acids across the human placenta: a review. Progress in Lipid Research 48 52-61. (doi:10.1016/j.plipres. 2008.11.001)

Elias SL \& Innis SM 2001 Infant plasma trans, n-6, and n-3 fatty acids and conjugated linoleic acids are related to maternal plasma fatty acids, length of gestation, and birth weight and length. American Journal of Clinical Nutrition 73 807-814.

Franke C, Demmelmair H, Decsi T, Campoy C, Cruz M, Molina-Font JA, Mueller K \& Koletzko B 2010 Influence of fish oil or folate supplementation on the time course of plasma redox markers during pregnancy. British Journal of Nutrition 103 1648-1656. (doi:10.1017/S0007114 509993746)

Frohnert B, Hui T \& Bernlohr D 1999 Identification of a functional peroxisome proliferator-responsive element in the murine fatty acid transport protein gene. Journal of Biological Chemistry 274 3970-3977. (doi:10.1074/jbc.274.7.3970)

Gil-Sánchez A, Larqué E, Demmelmair H, Acien MI, Faber FL, Parrilla JJ \& Koletzko B 2010 Maternal-fetal in vivo transfer of $\left[{ }^{13} \mathrm{C}\right]$ docosahexaenoic and other fatty acids across the human placenta $12 \mathrm{~h}$ after maternal oral intake. American Journal of Clinical Nutrition 92 115-122. (doi:10.3945/ajcn.2010.29589)

Gil-Sánchez A, Demmelmair H, Parrilla JJ, Koletzko B \& Larqué E 2011 Mechanisms involved in the selective transfer of long chain polyunsaturated fatty acids to the fetus. Frontiers in Genetics 2 57. (doi:10. 3389/fgene.2011.00057)

Haggarty P 2004 Effect of placental function on fatty acid requirements during pregnancy. European Journal of Clinical Nutrition 58 1559-1570. (doi:10.1038/sj.ejcn.1602016)

Haggarty P 2010 Fatty acid supply to the human fetus. Annual Review of Nutrition 30 237-255. (doi:10.1146/annurev.nutr.012809.104742)

Hanebutt FL, Demmelmair H, SchiessI B, Larqué E \& Koletzko B 2008 Longchain polyunsaturated fatty acid (LC-PUFA) transfer across the placenta. Clinical Nutrition 27 685-693. (doi:10.1016/j.clnu.2008.05.010)

Hatakeyama Y \& Scarpace PJ 2001 Transcriptional regulation of uncoupling protein-2 gene expression in L6 myotubes. International Journal of Obesity 25 1619-1624. (doi:10.1038/sj.ijo.0801812)

Hertelendy F \& Zakár T 2004 Prostaglandins and the myometrium and cervix. Prostaglandins, Leukotrienes, and Essential Fatty Acids 70 207-222. (doi:10.1016/j.plefa.2003.04.009)

Hewitt DP, Mark PJ \& Waddell BJ 2006a Placental expression of peroxisome proliferator-activated receptors in rat pregnancy and the effect of increased glucocorticoid exposure. Biology of Reproduction 74 23-28. (doi:10.1095/biolreprod.105.045914)

Hewitt DP, Mark PJ \& Waddell BJ 2006b Glucocorticoids prevent the normal increase in placental vascular endothelial growth factor expression and placental vascularity during late pregnancy in the rat. Endocrinology 147 5568-5574. (doi:10.1210/en.2006-0825)

Hoggard N, Crabtree J, Allstaff S, Abramovich DR \& Haggarty P 2001 Leptin secretion to both the maternal and fetal circulation in the ex vivo perfused human term placenta. Placenta 22 347-352. (doi:10.1053/plac. 2001.0628) 
Hun CS, Hasegawa K, Kawabata T, Kato M, Shimokawa T \& Kagawa Y 1999 Increased uncoupling protein2 mRNA in white adipose tissue, and decrease in leptin, visceral fat, blood glucose, and cholesterol in KK-Ay mice fed with eicosapentaenoic and docosahexaenoic acids in addition to linolenic acid. Biochemical and Biophysical Research Communications 259 85-90. (doi:10.1006/bbrc.1999.0733)

Imhoff-Kunsch B, Briggs V, Goldenberg T \& Ramakrishnan U 2012 Effect of $n-3$ long-chain polyunsaturated fatty acid intake during pregnancy on maternal, infant, and child health outcomes: a systematic review. Paediatric and Perinatal Epidemiology 26 91-107. (doi:10.1111/ j.1365-3016.2012.01292.x)

Jauniaux E, Watson AL, Hempstock J, Bao Y-P, Skepper JN \& Burton GJ 2000 Onset of maternal arterial blood flow and placental oxidative stress: a possible factor in human early pregnancy failure. American Journal of Pathology 157 2111-2122. (doi:10.1016/S0002-9440(10)64849-3)

Jauniaux E, Poston L \& Burton GJ 2006 Placental-related diseases of pregnancy: involvement of oxidative stress and implications in human evolution. Human Reproduction Update 12 747-755. (doi:10.1093/ humupd/dml016)

Jawerbaum A \& Capobianco E 2011 Review: Effects of PPAR activation in the placenta and the fetus: implications in maternal diabetes. Placenta 32 S212-S217. (doi:10.1016/j.placenta.2010.12.002)

Johnsen GM, Weedon-Fekjaer MS, Tobin KAR, Staff AC \& Duttaroy AK 2009 Long-chain polyunsaturated fatty acids stimulate cellular fatty acid uptake in human placental choriocarcinoma (bewo) cells. Placenta $\mathbf{3 0}$ 1037-1044. (doi:10.1016/j.placenta.2009.10.004)

Johnsen GM, Basak S, Weedon-Fekjær MS, Staff AC \& Duttaroy AK 2011 Docosahexaenoic acid stimulates tube formation in first trimester trophoblast cells, HTR8/SVneo. Placenta 32 626-632. (doi:10.1016/ j.placenta.2011.06.009)

Jones ML, Mark PJ, Lewis JL, Mori TA, Keelan JA \& Waddell BJ 2010 Antioxidant defenses in the rat placenta in late gestation: increased labyrinthine expression of superoxide dismutases, glutathione peroxidase 3, and uncoupling protein 2. Biology of Reproduction 83 254-260. (doi:10.1095/biolreprod.110.083907)

Jones ML, Mark PJ, Mori TA, Keelan JA \& Waddell BJ 2013a Maternal dietary omega-3 fatty acid supplementation reduces placental oxidative stress and increases fetal and placental growth in the rat. Biology of Reproduction 88 37. (doi:10.1095/biolreprod.112.103754)

Jones ML, Mark PJ, Keelan JA, Barden A, Mas E, Mori TA \& Waddell BJ 2013b Maternal dietary omega-3 fatty acid intake increases resolvin and protectin levels in the rat placenta. Journal of Lipid Research $\mathbf{5 4}$ 2247-2254. (doi:10.1194/jlr.M039842)

Jones ML, Mark PJ \& Waddell BJ 2013c Maternal omega-3 fatty acid intake increases placental labyrinthine antioxidant capacity, but does not protect against fetal growth restriction induced by placental ischemia-reperfusion injury. Reproduction 146 539-547. (doi:10.1530/ REP-13-0282)

Kang JX \& Lui A 2013 The role of the tissue omega-6/omega-3 fatty acid ratio in regulating tumor angiogenesis. Cancer Metastasis Review 32 201-210. (doi:10.1007/s10555-012-9401-9)

Keelan JA, Blumenstein M, Helliwell RJ, Sato TA, Marvin KW \& Mitchell MD 2003 Cytokines, prostaglandins and parturition - a review. Placenta 24 S33-S46. (doi:10.1053/plac.2002.0948)

Kemp MW, Saito M, Newnham JP, Nitsos I, Okamura K \& Kallapur SG 2010 Preterm birth, infection, and inflammation advances from the study of animal models. Reproductive Sciences 17 619-628. (doi:10.1177/ 1933719110373148)

Kuzmicki M, Telejko B, Szamatowicz J, Zonenberg A, Nikolajuk A, Kretowski A \& Gorska M 2009 High resistin and interleukin-6 levels are associated with gestational diabetes mellitus. Gynecological Endocrinology 25 258-263. (doi:10.1080/09513590802653825)

Kwak-Kim J, Yang KM \& Gilman-Sachs A 2009 Recurrent pregnancy loss: a disease of inflammation and coagulation. Journal of Obstetrics and Gynaecology Research 35 609-622. (doi:10.1111/j.1447-0756.2009. 01079.x)

Larqué E, Krauss-Etschmann S, Campoy C, Hartl D, Linde J, Klingler M, Demmelmair H, Caño A, Gil A, Bondy B et al. 2006 Docosahexaenoic acid supply in pregnancy affects placental expression of fatty acid transport proteins. American Journal of Clinical Nutrition 84 853-861.
Larqué E, Gil-Sánchez A, Prieto-Sanchez MT \& Koletzko B 2012 Omega 3 fatty acids, gestation and pregnancy outcomes. British Journal of Nutrition 107 S77-S84. (doi:10.1017/S0007114512001481)

Lepercq J, Challier JC, Guerre-Millo MI, Cauzac MI, Vidal H \& Hauguelde Mouzon S 2001 Prenatal leptin production: evidence that fetal adipose tissue produces leptin. Journal of Clinical Endocrinology and Metabolism 86 2409-2413. (doi:10.1210/jcem.86.6.7529)

Lin F, Zeng P, Xu Z, Ye D, Yu X, Wang N, Tang J, Zhou Y \& Huang Y 2012 Treatment of lipoxin A4 and its analogue on low-dose endotoxin induced preeclampsia in rat and possible mechanisms. Reproductive Toxicology 34 677-685. (doi:10.1016/j.reprotox.2012.09.009)

Linnemann K, Malek A, Sager R, Blum WF, Schneider H \& Fusch C 2000 Leptin production and release in the dually in vitro perfused human placenta. Journal of Clinical Endocrinology and Metabolism 85 4298-4301. (doi:10.1210/jcem.85.11.6933)

Magnusson AL, Waterman IJ, Wennergren M, Jansson T \& Powell TL 2004 Triglyceride hydrolase activities and expression of fatty acid binding proteins in the human placenta in pregnancies complicated by intrauterine growth restriction and diabetes. Journal of Clinical Endocrinology and Metabolism 89 4607-4614. (doi:10.1210/jc.2003032234)

Mailloux RJ \& Harper ME 2011 Uncoupling proteins and the control of mitochondrial reactive oxygen species production. Free Radical Biology \& Medicine 51 1106-1115. (doi:10.1016/j.freeradbiomed.2011.06.022)

Mark PJ, Lewis JL, Jones ML, Keelan JA \& Waddell BJ 2013 The inflammatory state of the rat placenta increases in late gestation and is further enhanced by glucocorticoids in the labyrinth zone. Placenta 34 559-566. (doi:10.1016/j.placenta.2013.04.006)

Massaro M, Scoditti E, Carluccio MA, Montinari MR \& De Caterina R 2008 Omega-3 fatty acids, inflammation and angiogenesis: nutrigenomic effects as an explanation for anti-atherogenic and anti-inflammatory effects of fish and fish oils. Journal of Nutrigenetics and Nutrigenomics 1 4-23. (doi:10.1159/000109871)

McCowen KC \& Bistrian BR 2005 Essential fatty acids and their derivatives. Current Opinion in Gastroenterology 21 207-215. (doi:10.1097/ 01.mog.0000153361.90653.cb)

Oh DY, Talukdar S, Bae EJ, Imamura T, Morinaga H, Fan W, Li P, Lu WJ, Watkins SM \& Olefsky JM 2010 GPR120 Is an omega-3 fatty acid receptor mediating potential anti-inflammatory and insulin-sensitizing effects. Cell 142 687-698. (doi:10.1016/j.cell.2010.07.041)

Oken E, Ning Y, Rifas-Shiman SL, Rich-Edwards JW, Olsen SF \& Gillman MW 2007 Diet during pregnancy and risk of preeclampsia or gestational hypertension. Annals of Epidemiology 17 663-668. (doi:10.1016/j.annepidem.2007.03.003)

Parsons JA, Brelje TC \& Sorenson RL 1992 Adaptation of islets of Langerhans to pregnancy: increased islet cell proliferation and insulin secretion correlates with the onset of placental lactogen secretion. Endocrinology 130 1459-1466. (doi:10.1210/endo.130.3.1537300)

Paulesu L, Bhattacharjee J, Bechi N, Romagnoli R, Jantra S \& letta F 2010 Pro-inflammatory cytokines in animal and human gestation. Current Pharmaceutical Design 16 3601-3615. (doi:10.2174/138161210793 797933)

Perkins AV 2006 Endogenous anti-oxidants in pregnancy and preeclampsia. Australian \& New Zealand Journal of Obstetrics \& Gynaecology 46 77-83. (doi:10.1111/j.1479-828X.2006.00532.x)

Prescott SL, Barden AE, Mori TA \& Dunstan JA 2007 Maternal fish oil supplementation in pregnancy modifies neonatal leukotriene production by cord-blood-derived neutrophils. Clinical Science 113 409-416. (doi:10.1042/CS20070111)

Recchiuti A \& Serhan CN 2012 Pro-resolving lipid mediators (SPMs) and their actions in regulating miRNA in novel resolution circuits in inflammation. Frontiers in Immunology 3 298. (doi:10.3389/fimmu. 2012.00298)

Richieri GV, Ogata RT, Zimmerman AW, Veerkamp JH \& Kleinfeld AM 2000 Fatty acid binding proteins from different tissues show distinct patterns of fatty acid interactions. Biochemistry 39 7197-7204. (doi:10.1021/bi000314z)

Rogers LK, Valentine CJ \& Keim SA 2013 DHA supplementation: current implications in pregnancy and childhood. Pharmacological Research 70 13-19. (doi:10.1016/j.phrs.2012.12.003) 
Rusterholz C, Hahn S \& Holzgreve W 2007 Role of placentally produced inflammatory and regulatory cytokines in pregnancy and the etiology of preeclampsia. Seminars in Immunopathology 29 151-162. (doi:10.1007/s00281-007-0071-6)

Ryan A \& Godson C 2010 Lipoxins: regulators of resolution. Current Opinion in Pharmacology 10 166-172. (doi:10.1016/j.coph.2010.02.005)

Schachtrup C, Emmler T, Bleck B, Sandqvist A \& Spener F 2004 Functional analysis of peroxisome-proliferator-responsive element motifs in genes of fatty acid-binding proteins. Biochemical Journal 382 239-245. (doi:10.1042/BJ20031340)

Schaiff WT, Knapp FF, Barak Y, Biron-Shental T, Nelson DM \& Sadovsky Y 2007 Ligand-activated peroxisome proliferator activated receptor $\gamma$ alters placental morphology and placental fatty acid uptake in mice. Endocrinology 148 3625-3634. (doi:10.1159/000327785)

Shoji H, Franke C, Demmelmair H \& Koletzko B 2009 Effect of docosahexaenoic acid on oxidative stress in placental trophoblast cells. Early Human Development 85 433-437. (doi:10.1016/ j.earlhumdev.2009.02.003)

Simopoulos AP 2011 Importance of the omega-6/omega-3 balance in health and disease: evolutionary aspects of diet. World Review of Nutrition and Dietetics 102 10-21. (doi:10.1159/000327785)

Stark MJ, Hodyl NA, Butler M \& Clifton VL 2012 Localisation and characterisation of uncoupling protein-2 (UCP2) in the human preterm placenta. Placenta 33 1020-1025. (doi:10.1016/j.placenta. 2012.09.010)

Stark M, Clifton V \& Hodyl N 2013 Differential effects of docosahexanoic acid (DHA) on preterm and term placental pro-oxidant/anti-oxidant balance. Reproduction 146 243-251. (doi:10.1530/REP-13-0239)
Umekawa T, Sugiyama T, Kihira T, Murabayashi N, Zhang L, Nagao K, Kamimoto Y, Ma N, Yodoi J \& Sagawa N 2008 Overexpression of thioredoxin-1 reduces oxidative stress in the placenta of transgenic mice and promotes fetal growth via glucose metabolism. Endocrinology 149 3980-3988. (doi:10.1210/en.2007-1682)

Vandevyver S, Dejager L, Tuckermann J \& Libert C 2013 New insights into the anti-inflammatory mechanisms of glucocorticoids: an emerging role for glucocorticoid-receptor-mediated transactivation. Endocrinology 154 993-1007. (doi:10.1210/en.2012-2045)

Wadhwani NS, Dangat KD, Joshi AA \& Joshi SR 2013 Maternal micronutrients and omega 3 fatty acids affect placental fatty acid desaturases and transport proteins in Wistar rats. Prostaglandins, Leukotrienes, and Essential Fatty Acids 88 235-242. (doi:10.1016/j.plefa.2012. 12.002)

World Health Organization 2011 Marine oil supplementation during pregnancy. Available from: http://www.who.int/elena/titles/fish_oil_ pregnancy/en/. [9 November 2012].

Wyrwoll CS, Mark PJ \& Waddell BJ 2005 Directional secretion and transport of leptin and expression of leptin receptor isoforms in human placental BeWo cells. Molecular and Cellular Endocrinology 241 73-79. (doi:10.1016/j.mce.2005.05.003)

Received 13 August 2013

First decision 14 October 2013

Revised manuscript received 23 December 2013

Accepted 21 January 2014 